\title{
Prevenção do suicídio no ambiente virtual: estratégias de divulgação e métricas de
}

\section{acessos de um website}

\author{
Suicide prevention in the virtual environment: strategies for dissemination and metrics for a \\ website
}

Prevención del suicidio en el entorno virtual: estrategias de divulgación y métricas de acceso para

un sitio web

Recebido: 06/12/2021 | Revisado: 17/12/2021 | Aceito: 24/12/2021 | Publicado: 26/12/2021

Camila Corrêa Matias Pereira

ORCID: https://orcid.org/0000-0001-6910-4148

Universidade de São Paulo, Brasil

E-mail:milamatias@hotmail.com

Daniele Maria Nogueira

ORCID: https://orcid.org/0000-0001-8442-9717

Universidade de São Paulo, Brasil

E-mail: dmnogueira27@gmail.com

Aline Conceição Silva

ORCID: https://orcid.org/0000-0001-5843-2517

Universidade de São Paulo, Brasil

E-mail: csilvaaline@usp.br

Laysa Fernanda Silva Pedrollo

ORCID: https://orcid.org/0000-0002-0489-7244

Universidade de São Paulo, Brasil

E-mail: laysa.pedrollo@usp.br

Bruna Marques Chiarelo

ORCID: https://orcid.org/0000-0001-8396-8874

Universidade de São Paulo, Brasil

E-mail: bruna.chiarelo@usp.br

Adriana Inocenti Miasso

ORCID: https://orcid.org/0000-0003-1726-7169

Universidade de São Paulo, Brasil

E-mail: amiasso@eerp.usp.br

Kelly Graziani Giacchero Vedana

ORCID: https://orcid.org/0000-0001-7363-2429

Universidade de São Paulo, Brasil

E-mail: kellygiacchero@eerp.usp.br

\begin{abstract}
Resumo
O suicídio é um comportamento multifatorial e um grave problema de saúde pública, sendo o ambiente virtual uma importante ferramenta para atuar na sua prevenção. Objetivo: Analisar as associações entre as estratégias de divulgação e os acessos diários de um website sobre a prevenção do comportamento suicida. Metodologia: Estudo quantitativo desenvolvido de 2019 a 2020 com o website InspirAção. Os dados do estudo foram coletados através do Google Analytics, redes sociais virtuais (Facebook e Instagram), jornais, registros de palestras e analisados por meio de estatística descritiva, teste de Mann-Whitney e Correlação de Spearman. Resultados: Todos os meios de divulgação estiveram associados a uma maior quantidade de sessões, novos usuários, visualizações de páginas e menor taxa de rejeição de acessos. Conclusão: As estratégias de divulgação de baixo custo são promissoras para promover o acesso a recursos para a prevenção do suicídio e para a propagação de conhecimentos científicos de forma compreensível e acessível.
\end{abstract}

Palavras-chave: Suicídio; Redes Sociais Online; Saúde Mental; Comunicação e Divulgação Científica.

\begin{abstract}
Suicide is a multifactorial behavior and is a public health problem. The virtual environment is a relevant strategie to prevent suicide. Aim: The aim of the project was to analyze associations between strategies for dissemination and daily accesses to a website for prevention of suicidal behavior. Methods: A Quantitative study was developed from 2019 to 2020 with the InspirAção's website. The data collection was made through Google Analytics, social media platforms (Facebook and Instagram), newspapers and lectures records. The data was analyzed using descriptive statistics, Mann-Whitney test and Spearman correlation. Results: The strategies of dissemination were associated with
\end{abstract}


a greater number of access, new users, page views and a lower rejection rate. Conclusions: Low-cost dissemination strategies are promising to promote access to resources for suicide prevention and to disseminate scientific knowledge in an understandable and accessible way.

Keywords: Suicide; Online Social Networking; Mental Health; Scientific Communication and Diffusion.

\section{Resumen}

El suicidio es un comportamiento multifactorial y un grave problema de salud y el entorno virtual es una herramienta importante para actuar en su prevención. Objetivo: Analizar las asociaciones entre las estrategias de difusión y los accesos diarios a un sitio web para la prevención de conductas suicidas. Metodología: Estudio cuantitativo desarrollado de 2019 a 2020 con el sitio web InspirAção. Los datos del estudio fueron recolectados por Google Analytics, redes sociales (Facebook e Instagram), periódicos y registros de conferencias y analizados mediante estadística descriptiva, prueba de Mann-Whitney y correlación de Spearman. Resultados: Todos los medios de difusión se asociaron a un mayor número de sesiones, nuevos usuarios, páginas vistas y una menor tasa de rechazo de visitas. Conclusiones: Las estrategias de difusión de bajo costo son prometedoras para promover el acceso a los recursos para la prevención del suicidio y para difundir el conocimiento científico de una manera comprensible y accesible.

Palabras clave: Suicidio; Redes Sociales en Línea; Salud Mental; Comunicación y Divulgación Científica.

\section{Introdução}

O suicídio é um comportamento multifatorial que gera impactos significativos a nível mundial, sendo um grave problema de saúde pública. Estima-se que a cada 40 segundos uma pessoa morra por suicídio, totalizando aproximadamente 700.000 mortes todos os anos (Who, 2019). Por se tratar de um fenômeno complexo, precisa ser investigado em diversos públicos, contextos e ambientes, inclusive os virtuais (Starcevic \& Aboujaoude, 2015). O ambiente virtual é uma importante ferramenta de comunicação e expressão de sentimentos, podendo envolver a busca de conteúdos preventivos e pró-suicidas (Wongkoblap, Vadillo \& Curcin, 2017).

A relação entre os ambientes virtuais e os fatores de risco e proteção contra o suicídio vêm progressivamente ganhando espaço no meio científico (Belfort \& Miller, 2018; Dodemaide, Joubert, Merolli, \& Hill, 2019; Marchant et al., 2017; Robert, Suelves, Armayones, \& Ashley, 2015). O contato com as atividades em ambiente virtual pode resultar em efeitos positivos ou negativos sobre a saúde mental dos indivíduos que se encontram em situação de vulnerabilidade, visto que o ambiente virtual pode oportunizar aos usuários acesso a conteúdos protetivos contra o suicídio, mas também apresentar vivências nocivas, permeadas por fatores de risco (Robert, Suelves, Armayones, \& Ashley, 2015). Dessa forma, é importante entender como a Internet pode encorajar ou prevenir o comportamento suicida (Krysinska et al., 2017).

Indivíduos emocionalmente vulneráveis podem buscar conteúdos relacionados à temática, podendo encontrar materiais pró-suicidas no ambiente virtual (Belfort \& Miller, 2018; Dodemaide, Joubert, Merolli, \& Hill, 2019; Marchant et al., 2017). Quando se trata do comportamento suicida existe também a preocupação com o "Efeito Contágio" ou "Efeito Werther", pela propagação do tema de forma irresponsável, com suicídios amplamente divulgados e reação aos temas considerados de risco para aqueles que estão em sofrimento emocional (Carmichael \& Whitley, 2019).

Nos últimos anos, os ambientes virtuais tem proporcionado espaço para o desenvolvimento de iniciativas, tecnologias e recursos virtuais que podem ser importantes ferramentas aliadas a prevenção do suicídio. Entretanto, ao analisar a literatura sobre essa temática é possível observar que a comprovação científica em relação a esses produtos virtuais ainda é escassa (Belfort \& Miller; Dodemaide et al., 2019; Franco-Martín et al., 2018; Kreuze et al., 2017). No Brasil, recentes revisões de literatura não identificaram publicações sobre websites ou aplicativos brasileiros sobre a prevenção do suicídio (Marchant et al., 2017; Platts \& Morgan, 2018; Lopez-Castroman et al., 2020).

Há a necessidade de utilizar iniciativas, tecnologias e recursos virtuais seguros e embasados cientificamente para a prevenção do comportamento suicida (Franco-Martín et al., 2018; Platts \& Morgan, 2018). As potencialidades do ambiente virtual podem colaborar com o planejamento de ações relacionadas à disseminação de informações de suporte e de uso seguro 
do meio virtual, bem como a desestigmatização e fortalecimento de fatores protetivos relacionados aos comportamentos em saúde da população (Notredame et al., 2018). Além disso, esse meio apresenta potencial para contribuir em processos com foco na formação e capacitação de recursos humanos para área da saúde (Notredame et al., 2018).

Os avanços no meio virtual também têm proporcionado novas visões em relação aos processos de divulgação e disseminação de conteúdos sobre a prevenção do suicídio, principalmente no que se refere a utilização de estratégias de divulgação sustentáveis como forma de viabilizar o acesso às informações e conteúdos científicos seguros (Notredame et al., 2018). A compreensão de como a prevenção do suicídio tem sido realizada em meios virtuais de forma segura e permeada por conhecimento científico pode fornecer subsídios que contribuam com o desenvolvimento, acompanhamento e avaliação de novas iniciativas virtuais nessa área.

Dessa forma, o presente estudo teve como objetivo analisar as associações entre as estratégias de divulgação científica e as métricas de acesso de um website brasileiro voltado para a prevenção do comportamento suicida.

\section{Metodologia}

\section{Tipo de Estudo}

Estudo transversal com abordagem quantitativa, desenvolvido a partir da coleta de dados sobre métricas de acesso e estratégias de divulgação do website brasileiro para prevenção do comportamento suicida. Na pesquisa transversal, o fator e o efeito são observados no mesmo momento histórico (Rouquayrol, 1993) e a pesquisa qualitativa permite a construção de conhecimento através de opiniões, valores, crenças, relações, representações e ações humanas levando em consideração toda a intersubjetividade dos achados (Minayo, 2012).

\section{Cenário}

O website analisado se trata de uma iniciativa científica brasileira voltada para a divulgação científica relacionada a promoção da saúde mental e prevenção do suicídio no ambiente virtual. Assim, busca atender recomendações internacionais sobre prevenção virtual do suicídio, tais como a intervenção comunitária, cientificamente fundamentada, segura, acessível, com proteção de anonimato e com enfoque nos fatores de risco e protetivos contra o suicídio (Kreuze et al., 2017; Platts \& Morgan, 2018).

O InspirAção possibilita o acesso a conteúdos científicos desenvolvidos pelo Laboratório de Estudos e Pesquisa em Prevenção e Posvenção do Suicídio (LEPS), dentre eles, artigos científicos, materiais educativos (livros, cartilhas, vídeos, podcasts), postagens de especialistas, e ferramentas virtuais de promoção de saúde mental.

Desenvolve-se enquanto uma atividade de extensão do Centro de Educação em Prevenção e Posvenção do Suicídio (CEPS) da Escola de Enfermagem de Ribeirão Preto da Universidade de São Paulo (EERP-USP) para popularização científica reforçando o compromisso social de professores e estudantes de graduação e pós-graduação de uma instituição de ensino pública. O InspirAção não tem a finalidade de oferecer atendimento virtual, mas colaborar para a promoção e fortalecimento de estratégias de cuidado.

\section{Estratégias de Divulgação}

Para a divulgação científica inicial foram elencadas estratégias como publicações sobre o website no Facebook oficial do grupo de pesquisa (com 4.405 seguidores), postagens no Instagram oficial do InspirAção (com 885 seguidores no período), palestras educativas com divulgação do website realizadas por membros do CEPS/LEPS, reportagens em mídias jornalísticas, além de outras colaborações (influenciadores digitais, instituições de ensino e grupos de pesquisa). No período de coleta não 
foram realizadas contratações de anúncios publicitários sobre o InspirAçãoß. Todas as estratégias desenvolvidas foram sistematicamente documentadas em banco de dados para possibilitar as análises estatísticas do estudo.

\section{Medidas}

A coleta das métricas de acesso do website foi realizada diariamente por meio de informações disponibilizadas pelo serviço Google Analytics. O serviço é oferecido de forma gratuita pelo Google $\mathbb{R}$ e tem por objetivo a exibição de estatísticas relacionadas ao acesso de websites. Para este estudo foram utilizadas as informações sobre os países com mais acessos, quantidade usuários que visitaram o website pela primeira vez (novos usuários), quantidade de sessões (número de visitas que o website recebeu), tempo que os usuários permaneceram no website, número total de páginas visualizadas e taxas de rejeição de acessos (percentual de sessões de uma única página).

\section{Coleta, Tratamento e Análise dos Dados}

A coleta de dados foi realizada durante seis meses após o lançamento do website (1 de setembro 2019 a 29 de fevereiro de 2020), utilizando instrumento criado pelas pesquisadoras com as variáveis analisadas, havendo a coleta de dados diariamente (número de sessões, tempo de duração em segundos, taxa de rejeição de acessos, tipo de dispositivo, procedência país, procedência cidade, conteúdo, visualizações da página, novos usuários e se no dia havia publicações no Instagram e Facebook do grupo de pesquisa, palestras ministradas por membros ou reportagens). Vale frisar, que os 10 dias que obtiveram mais acessos foram submetidas a análises que serão discutidas posteriormente neste artigo. $\mathrm{O}$ roteiro mensal era composto por: procedência país e cidade, páginas mais acessadas, origem/mídia de acesso ao site, tipos de aparelho, referências e número de usuários. Esse processo foi realizado com apoio de profissionais especializados na área de Tecnologia da Informação. Os dados foram digitados e tratados em uma planilha e transportados para o Statistical Package for the Social Sciences (SPSS) versão 21.0 (Pallant, 2010).

Inicialmente, os dados foram analisados por estatística descritiva. Foram utilizados os testes Kolmogorov-Smirnov e Shapiro-Wilk para avaliar a normalidade da distribuição das variáveis quantitativas. Apenas a taxa de rejeição de acessos teve distribuição normal. Em seguida, foram empregados o Teste $\mathrm{t}$ (para comparação de médias relacionadas às taxas de rejeição de acessos), o teste de Mann-Whitney (para comparação de medianas relacionadas às quantidades de sessões, duração das sessões, novos usuários e visualizações de páginas) e o teste de Correlação de Spearman (para avaliar a correlação entre quantidade de novos usuários, tempo de duração das sessões e taxa de rejeição de acessos).

\section{Aspectos Éticos}

O estudo obteve aprovação do Comitê de Ética em Pesquisa sendo desenvolvido em concordância com as recomendações de pesquisa envolvendo seres humanos (CNS 466/2012). Destaca-se que a visualização do InspirAção não requer cadastro do usuário, porém, este é necessário para o envio de conteúdos ao website. O cadastramento pode ser realizado apenas por pessoas com idade igual ou superior a 18 anos sendo acompanhado pelo aceite dos termos de uso do website e do Termo de Consentimento Livre e Esclarecido (TCLE) relacionado à pesquisa.

\section{Resultados}

Durante os seis meses após o seu lançamento, o website brasileiro para prevenção do comportamento suicida obteve o total de 64.628 visualizações, realizadas por 12.997 usuários em 18.161 sessões. O website foi acessado em 26 países diferentes, sendo que Brasil (94,94\%), Portugal (1,68\%) e os Estados Unidos (1,66\%), representaram, respectivamente, os três 
primeiros países com mais acessos. Em relação ao idioma dos usuários, o português do Brasil (90,63\%) foi predominante, seguido pelo inglês $(5,17 \%)$ e português de Portugal $(2,25 \%)$.

No período analisado as estratégias de divulgação do website totalizaram $n=355$, sendo representadas por 20 reportagens em mídias jornalísticas, 62 palestras educativas, 138 publicações no Facebook do grupo de pesquisa da qual as autoras fazem parte (com 3.878 curtidas, 1.553 compartilhamentos e 158 comentários), 63 postagens no Instagram oficial do website (com 1991 likes e 48 comentários) e 72 contatos diretos com influenciadores digitais, instituições de ensino e grupos de pesquisa. Todas as informações sobre o website produzidas em postagens, textos, infográficos e materiais complementares para a divulgação foram previamente elaboradas e revisadas por membros do grupo de pesquisa. As postagens de divulgação priorizavam a disseminação de informações sucintas sobre a temática abordada no website, bem como a disponibilização da logomarca e hiperlink de redirecionamento para o acesso aos conteúdos e objetivo da iniciativa.

Dentre as mídias jornalísticas analisadas e envolvidas na divulgação do website estiveram jornais virtuais brasileiros de grande circulação, mídias ligadas à área acadêmica e também conselhos profissionais, tais como: Jornal da USP

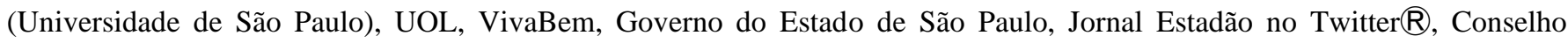
Regional de Enfermagem de São Paulo (Coren-SP) e Conselho Regional de Psicologia de São Paulo (CRP-SP).

Considerando os 10 dias analisados que obtiveram a maior parte dos acessos, sendo o número de acessos $(47,3 \%)$, maioria dos novos usuários $(53,9 \%)$ e das visualizações das páginas $(50,5 \%)$ referentes aos seis meses de dados coletados, nota-se que neste período, as palestras que divulgaram o website foram realizadas em cinco dias. As publicações em mídias jornalísticas sobre o website ocorreram em sete dias dos 10 analisados, sendo que nos três dias restantes foram precedidos por uma estratégia de divulgação do website (chamada de "Dia D" e elaborada pelo grupo de pesquisa), para influenciadores digitais e grupos envolvidos com causas sociais que pudessem ter interesse em colaborar com a divulgação. É importante ressaltar que os três dias analisados acima ocorreram no mês de setembro, mês conhecido pelo "Setembro Amarelo", dedicado à prevenção e conscientização contra o suicídio, tendo, portanto, os objetivos alinhados com o conteúdo do website.

A realização de palestras com a divulgação do website foram associadas a maiores quantidades de sessões, novos usuários, maior visualizações de páginas, maior duração das sessões e menor taxa de rejeição de acessos. As reportagens sobre o website estiveram associadas a maiores quantidades de sessões, novos usuários, maiores visualizações de páginas e menor taxa de rejeição de acessos. Destaca-se que nos dias em que ocorreram a divulgação do website por meio de reportagens, as medianas referentes às sessões, novos usuários e visualizações de página, foram de 13 a 20 vezes superiores aos valores obtidos nos dias sem reportagens (Tabela 1). 
Tabela 1 - Associações entre métricas de acesso do website InspirAção, palestras e reportagens sobre o website, no período de 01 de setembro 2019 a 29 de fevereiro de 2020.

\begin{tabular}{|c|c|c|c|c|c|c|}
\hline \multirow[t]{3}{*}{ Variáveis } & \multicolumn{2}{|c|}{ Palestras } & \multicolumn{4}{|c|}{ Reportagens } \\
\hline & Não & Sim & & Não & Sim & \\
\hline & Md (Int) & Md (Int) & $\mathrm{p}$ & Md (Int) & Md (Int) & $\mathrm{p}$ \\
\hline $\begin{array}{l}\text { Número de } \\
\text { sessões* }\end{array}$ & $26(3-846)$ & $76(16-1863)$ & $<0,001$ & $33(3-1050)$ & $435(80-1863)$ & $<0,001$ \\
\hline $\begin{array}{l}\text { Duração das } \\
\text { sessões (seg)* }\end{array}$ & $138(3-439)$ & $166(61-581)$ & 0,045 & $142(3-581)$ & $168(121-201)$ & 0,292 \\
\hline Novos usuários* & $14(2-786)$ & $33(6-1671)$ & $<0,001$ & $16(2-939)$ & $326(44-1671)$ & 0,002 \\
\hline & $386(7-2352)$ & $312(50-8431)$ & $<0,001$ & $112(7-3343)$ & $1703(356-8431)$ & $<0,001$ \\
\hline & $\mathrm{M}$ (DP) & $\mathrm{M}$ (DP) & $\mathrm{p}$ & $\mathrm{M}$ (DP) & M (DP) & $\mathrm{p}$ \\
\hline $\begin{array}{l}\text { Taxa de rejeição } \\
\text { de acessos** }\end{array}$ & 59(13) & $55(9)$ & 0,010 & $59(12)$ & $47(8)$ & 0,003 \\
\hline
\end{tabular}

Md: Mediana; Int: Intervalo; p: valor de p; * Teste adotado: Mann-Whitney; ** Teste adotado: teste t. Fonte: Os autores.

As publicações sobre o website em perfis do grupo de pesquisa no Instagram $\mathbb{R}$ e no Facebook $\mathbb{R}$ estiveram associadas a maiores quantidades de sessões, novos usuários, maiores visualizações de páginas e menor taxa de rejeição de acessos (Tabela 2). O teste de Spearman identificou que a maior quantidade de novos usuários esteve correlacionada a maior tempo de duração das sessões $(r=, 209 ; \mathrm{p}=0,005)$ e a menor taxa de rejeição de acessos $(r=-, 404 ; \mathrm{p}=<0,001)$.

Tabela 2 - Associações entre métricas de acesso do website InspirAção, publicações em Instagram e Facebook sobre o website no período de 01 de setembro 2019 a 29 de fevereiro de 2020.

\begin{tabular}{|c|c|c|c|c|c|c|}
\hline \multirow[t]{3}{*}{ Variáveis } & \multicolumn{2}{|c|}{ Instagram } & \multicolumn{4}{|c|}{ Facebook } \\
\hline & Não & Sim & & Não & Sim & \\
\hline & Md (Int) & Md (Int) & $\mathrm{p}$ & Md (Int) & Md (Int) & $\mathrm{p}$ \\
\hline $\begin{array}{l}\text { Número de } \\
\text { sessões* }\end{array}$ & $28(3-893)$ & $55(10-1863)$ & 0,003 & $22(3-435)$ & $59(10-1863)$ & $<0,001$ \\
\hline $\begin{array}{l}\text { Duração das } \\
\text { sessões (seg)* }\end{array}$ & $138(3-581)$ & $157(5-317)$ & 0,224 & $139(3-581)$ & $157(5-364)$ & 0,231 \\
\hline Novos usuários* & $16(2-786)$ & $24(4-1671)$ & $\mathbf{0 , 0 1 3}$ & $13(2-354)$ & $31(4-1671)$ & $<0,001$ \\
\hline \multirow[t]{2}{*}{$\begin{array}{l}\text { Visualizações da } \\
\text { página* }\end{array}$} & $97(7-3354)$ & $174(13-8431)$ & 0,005 & $77(7-1703)$ & $199(16-8431)$ & $<0,001$ \\
\hline & M (DP) & $\mathrm{M}(\mathrm{DP})$ & $\mathrm{p}$ & $\mathrm{M}(\mathrm{DP})$ & M (DP) & $\mathrm{p}$ \\
\hline $\begin{array}{l}\text { Taxa de rejeição } \\
\text { de acessos** }\end{array}$ & $60(13)$ & $52(10)$ & $\mathbf{0 , 0 1 7}$ & $60(14)$ & $56(10)$ & $\mathbf{0 , 0 3 1}$ \\
\hline
\end{tabular}

Md: Mediana; Int: Intervalo; p: valor de p; * Teste adotado: Mann-Whitney ; ** Teste adotado: teste t. Fonte: Os autores. 


\section{Discussão}

Este estudo identificou estratégias de divulgação como reportagens, palestras e publicações em redes sociais estiveram associadas a melhores métricas de acesso (maior quantidade de sessões, novos usuários, visualizações de páginas e menor taxa de rejeição de acessos) de um website brasileiro para prevenção do suicídio (InspirAção). Entre os diferentes métodos de divulgação, as palestras foram a única estratégia associada a maior duração dos acessos. Apesar de não haver na literatura elementos suficientes para explicar esse achado, é possível que o mesmo esteja relacionado ao tipo de interação (presencial ou offline), maior aproximação com o conteúdo do website e maior interesse instigado pelas palestras.

Os períodos com reportagens se destacaram por apresentar média de sessões, novos usuários e visualizações de 13 a 20 vezes maiores do que os períodos sem reportagens. Estudo australiano de divulgação de website para prevenção do suicídio masculino obteve resultados positivos de acesso durante exibição televisiva sobre website. Assim, destaca a possibilidade do uso do entretenimento para ampliar acesso a informações científicas sobre tópicos sensíveis em saúde e fornecer melhores intervenções de prevenção e suporte (King, Schlichthorst, Turnure, Phelps, Spittal \& Pirkis, 2019).

O contato de pesquisadores em suicidologia com profissionais da mídia, além de propiciar maior divulgação de recursos preventivos, pode oportunizar a educação em saúde mental, a adesão das mídias às recomendações da Organização Mundial da Saúde (OMS) relacionadas a divulgações de conteúdos sobre o suicídio (World Health Organization, 2008). Entretanto, pesquisa brasileira encontrou que as recomendações são parcialmente seguidas pela mídia jornalística e que a minoria das notícias divulga recursos de apoio (Ferreira, Martin, Zanetti \& Vedana, 2019). Dessa forma, indica a necessidade de ações educativas que incentivem o envolvimento seguro da mídia na divulgação de matérias que colaborem com a prevenção do suicídio evidenciando recursos e estratégias de apoio (Ferreira et al., 2019).

A maior quantidade de novos usuários esteve correlacionada com maior tempo de duração das sessões e a menor taxa de rejeição de acessos, evidenciando que o website pode ter sido mais atrativo para os novos usuários. A dificuldade de engajamento de usuários em websites de prevenção do suicídio pode ser uma barreira para as ações preventivas (LopezCastroman et al., 2020). Esse achado indica a necessidade de criação, dinamização no planejamento, atualização e divulgação de novos conteúdos, bem como a incorporação de habilidades e parcerias para promover o engajamento de usuários nos recursos preventivos em ambientes virtuais.

Evidências sugerem que várias estratégias e canais de comunicação em mídias virtuais têm sido utilizados para divulgar conhecimentos científicos sobre saúde, promover o autocuidado e a tomada de decisões políticas com maior embasamento e informações. Todavia, a comunicação científica por pesquisadores ainda é uma atividade desafiadora em expansão, visto que pesquisadores da saúde estão usualmente mais preparados e habituados a comunicar suas descobertas entre os pares do que engajar públicos mais amplos no meio digital, o que demanda habilidades e conhecimentos específicos (Fontaine et al., 2019).

A maioria das visualizações de páginas e dos novos usuários do website esteve concentrada em 10 dias (entre setembro e outubro), período no qual houve palestras, divulgação por influenciadores digitais e reportagens. Faz-se importante que essas formas de divulgação sejam realizadas de modo continuado para facilitar o acesso e consumo de conteúdos de apoio e formativos que possam contribuir, especialmente, com profissionais de saúde (Vedana, Magrini, Zanetti, Miasso, Borges, \& Santos, 2017) e pessoas em risco de suicídio e fontes de apoio para esses indivíduos.

As estratégias virtuais tem potencial para complementar outras modalidades de acompanhamento, dentre elas, o apoio, orientação e redução dos riscos relacionados ao comportamento suicida (Belfort \& Miller, 2018; Dodemaide et al., 2019; Franco-Martin et al., 2018; Kreuze et al., 2017; Platts \& Morgan, 2018). Assim, é importante que esses recursos sejam amplamente divulgados e aprimorados oportunamente a partir do engajamento dos usuários. 
As formas de divulgação empregadas no presente estudo são de baixo custo, relativamente sustentáveis, promissoras e acessíveis para grupos de pesquisa. As estratégias ainda mostram potencial de alcance para favorecer a prevenção do suicídio, uma vez que o engajamento de usuários em websites de prevenção seguros e baseados em evidências científicas é um importante desafio para a ampliação das ações preventivas (Notredame et al., 2018). Entretanto, apesar da crescente expansão dos recursos tecnológicos (blogs, websites, aplicativos, podcats, entre outros), essas atividades requerem avaliação de sua efetividade (Fontaine et al., 2019). Vale ressaltar ainda que revisões de literatura não têm encontrado produções brasileiras sobre o assunto (Marchant et al., 2017; Lopez-Castroman et al., 2020).

Dessa forma, é importante desenvolver estratégias de divulgação que permitam o acesso da população a fontes de informação e apoio idôneas, baseadas em evidências científicas e acessíveis no que tange ao suicídio, Nesse sentido, possibilita-se a popularização do conhecimento científico, favorecendo a aproximação entre a Universidade e sociedade, articulando de forma indissociável as atividades de pesquisa, ensino e extensão universitárias, além de contribuir para uma tomada de decisão segura em saúde.

\section{Considerações Finais}

O presente estudo realizou a análise de associações entre as estratégias de divulgação científica e as métricas de acesso de um website brasileiro voltado para a prevenção do comportamento suicida. As estratégias de divulgação analisadas referem-se a atividades de baixo custo e sustentáveis (reportagens, palestras e publicações em perfis de redes sociais) as quais estiveram associadas à melhores métricas de acesso de um website voltado para a prevenção do suicídio (maior quantidade de sessões, novos usuários, visualizações de páginas e menor taxa de rejeição de acessos). Tais estratégias podem ser promissoras para os recursos protetivos sobre a prevenção do suicídio, embora ainda escassos, sejam reconhecidos e acessados por pessoas que podem se beneficiar dos mesmos.

Além disso, as formas de divulgação mencionadas neste estudo podem fomentar e ser ponto de partida para atividades desenvolvidas por outras iniciativas virtuais que visam a propagação de conhecimentos científicos de forma segura, compreensível e acessível para a população geral. O compartilhamento de conhecimento científico através de canais confiáveis e vinculados a instituições de pesquisa pode ser um importante recurso para combater informações falsas e reduzir o preconceito e o estigma sobre a temática. Além de produzir conhecimento e encontrar novas estratégias para a prevenção considerando o ambiente virtual, é igualmente importante analisar as estratégias de divulgação e métricas de acessos dessas iniciativas com o objetivo de aprimorar iniciativas futuras voltadas para a prevenção em saúde. Ao considerar os avanços tecnológicos, as iniciativas virtuais tem potencial inovador e podem oferecer respaldo e informações que podem subsidiar as divulgações no ambiente virtual, bem como o desenvolvimento de futuras pesquisas na área, aumentando o conteúdo protetivo.

Dentre as limitações do estudo, destaca-se que os dados coletados e analisados representam um recorte de dias em relação às métricas e divulgação da iniciativa, dessa forma, estudos mais aprofundados podem apresentar olhares mais amplos sobre as influências da divulgação em relação às métricas existentes. Junto a isso, as divulgações foram realizadas em âmbito nacional, sem a utilização de recursos financeiros para o maior alcance de usuários nos ambientes virtuais.

\section{Agradecimentos}

O presente trabalho foi realizado com apoio e financiamento da Fundação de Amparo à Pesquisa do Estado de São Paulo (FAPESP) e da Coordenação de Aperfeiçoamento de Pessoal de Nível Superior Brasil (CAPES) - - Código de Financiamento 001. 


\section{Referências}

Belfort, E. L., \& Miller, L. (2018). Relationship between adolescent suicidality, self-injury, and media habits. Child and Adolescent Psychiatric Clinics of North America, 27(2), 159-169. doi: 10.1016/j.chc.2017.11.004

Carmichael, V., \& Whitley, R. (2019). Media coverage of Robin Williams' suicide in the United States: a contributor to contagion? PLOS ONE.14(5):e0216543. doi: 10.1371/journal.pone.0216543. eCollection 2019.

Dodemaide, P., Joubert, L., Merolli, M., \& Hill, N. (2019). Exploring the therapeutic and nontherapeutic affordances of social media use by young adults with lived experience of self-harm or suicidal ideation: a scoping review. Cyberpsychology, Behavior, and Social Networking, 22(10), 622-633. doi: $10.1089 /$ cyber.2018.0678

Ferreira, R. S., Martin, I.S., Zanetti, A.C.G., \& Vedana, K.G.G. (2019). Notícias sobre suicídio veiculadas em jornal brasileiro. Ciencia Saude Coletiva, 225. Disponível em: http://www.cienciaesaudecoletiva.com.br/artigos/noticias-sobre-suicidio-veiculadas-em-jornal-brasileiro/17299?id=17299

Fontaine, G., Maheu-Cadotte, M. A., Lavallée, A., Mailhot, T., Rouleau, G., Bouix-Picasso, J., \& Bourbonnais, A. (2019). Communicating science in the digital and social media ecosystem: scoping review and typology of strategies used by health scientists. JMIR Public Health Surveill, 5(3),e14447. doi: $10.2196 / 14447$

Franco-Martín, M. A. Muñoz-Sánchez, J. L., Sainz-de-Abajo, B., Castillo-Sánchez, G., Hamrioui, S., \& de la Torre-Díez, I. (2018). A systematic literature review of technologies for suicidal behavior prevention. Journal of Medical Systems, 42(4). doi: 10.1007/s10916-018-0926-5

King, K., Schlichthorst, M., Turnure, J., Phelps, A., Spittal, M. J., \& Pirkis, J. (2019). Evaluating the effectiveness of a website about masculinity and suicide to prompt help-seeking. Health promotion journal of Australia: official journal of Australian Association of Health Promotion Professionals, 30(3), 381-389. doi: 10.1002/hpja.237

Kreuze, E., Jenkins, C. Gregoski, M., York, J., Mueller, M., Lamis, D. A., \& Ruggiero, K. J. (2017). Technology-enhanced suicide prevention interventions: a systematic review. Journal of Telemedicine and Telecare, 23(6), 605-617. doi: 10.1177/1357633X16657928

Krysinska, K., Westerlund, M., Niederkrotenthaler, T., Andriessen, K., Carli, V., Hadlaczky, G., Till, B., \& Wasserman, D. (2017). A Mapping Study on the Internet and Suicide. Crisis, 38(4), 217-226. https://doi.org/10.1027/0227-5910/a000444

Lopez-Castroman, J., Moulahi, B., Azé, J. Bringay, S., Deninotti, J., Guillaume, S. \& Baca-Garcia, E. (2020). Mining social networks to improve suicide prevention: a scoping review. Journal of Neuroscience Research, 98(4),616-625. doi: 10.1002/jnr.24404

Marchant, A., Hawton, K., Stewart, A., Montgomery, P., Singaravelu, V., Lloyd, K., Purdy, N., Daine, K., \& John, A. (2017). A systematic review of the relationship between internet use, self- harm and suicidal behaviour in young people: the good, the bad and the unknown. PLoS One,12(8), e0181722. doi: 10.1371/journal.pone.0181722

Minayo, MCS. (2012). Análise qualitativa: teoria, passos e fidedignidade. Ciência \& Saúde Coletiva, 17(3), 621-626. doi:10.1590/S1413-81232012000300007

Notredame, C. E., Grandgenèvre, P., Pauwels, N., Morgiève, M., Wathelet, M., Vaiva, G., \& Séguin, M. (2018). Leveraging the web and social media to promote access to care among suicidal individuals. Frontiers in Psychology, 9, 1338 Disponível em: https://doi.org/10.3389/fpsyg.2018.01338

Pallant, J. (2016). SPSS survival manual: a step by step guide to data analysis using SPSS. (4.ed.), Open University Press.

Platts,D., \& Morgan, S. (2018). Comment on 'Web-based tools and mobile applications to mitigate burnout, depression, and suicidality among healthcare students and professionals: a systematic review. Academic psychiatry, 42(3), 422-423. doi: 10.1007/s40596-017-0868-0

Robert, A., Suelves, J. M., Armayones, M., \& Ashley, S. (2015). Internet use and suicidal behaviors: internet as a threat or opportunity?. TELEMEDICINE and e-HEALTH, 21(4):306-11. doi: 10.1089/tmj.2014.0129

Rouquayrol MZ. (1993). Epidemiologia e saúde. (4.ed.), Rio de Janeiro.

Starcevic, V., \& Aboujaoude, E. (2015). Cyberchondria, cyberbullying, cybersuicide, cybersex: "new" psychopathologies for the 21st century?. World Psychiatry, 14(1), 97-100. doi: 10.1002/wps.20195

Vedana K. G. G., Magrini, D. F., Zanetti, A. C. G., Miasso, A. I., Borges, T. L., \& Santos, M. A. (2017). Attitudes towards suicidal behaviour and associated factors among nursing professionals: a quantitative study. Journal of Psychiatric and Mental Health Nursing, 24(9-10), 651-659. doi: 10.1111/jpm.12413

Wongkoblap, A., Vadillo, M. A., \& Curcin, V. (2017). Mental health disorders in the era of social media: systematic review. Journal of Medical Internet Research, 19(6):e228. doi: 10.2196/jmir.7215

World Health Organization. (2008). Preventing Suicide: A resource for media professionals. Geneva: WHO. Disponível em: https://www.who.int/mental_health/suicide-prevention/resource_booklet_2017/en/

World Health Organization. (2019). Suicide in the world: global health estimates. Geneva: WHO. Disponível em: https://apps.who.int/iris/handle/10665/326948 